A Free Weekly Self-Administered at-Home Virtual Reality based

Protocol for Overcoming the Psychological Burden of Coronavirus:

A Pragmatic Waitlist-Controlled Trial Conducted During COVID-19 lockdown in Italy with 2-week follow-up

Giuseppe Riva (giuseppe.riva@unicatt.it) ${ }^{1-2}$, Luca Bernardelli (luca.bernardelli@become-hub.com) ${ }^{3}$, Gianluca Castelnuovo (gianluca.castelnuovo@unicatt.it) ${ }^{1-4}$, Alex Clementi

(alex.clementi01@icatt.it) ${ }^{2}$, Daniele Di Lernia

(daniele.dilernia@unicatt.it) ${ }^{2}$, Elisa Pedroli

(e.pedroli@auxologico.it) ${ }^{1-5}$, Clelia Malighetti

(clelia.malighetti@unicatt.it)2, Francesca Sforza

(francesca.sforza@become-hub.it) ${ }^{3}$, Brenda K. Wiederhold

(b@vrphobia.eu) ${ }^{6-7}$, Silvia Serino (silvia.serino@unicatt.it) ${ }^{*}$

1 IRCCS Istituto Auxologico Italiano, Milan, Italy

2 Humane Technology Lab., Università Cattolica del Sacro Cuore, Milan, Italy

${ }^{3}$ Become-Hub, Milan, Italy

${ }^{4}$ Department of Psychology, Università Cattolica del Sacro Cuore, Milan, Italy

${ }^{5}$ Faculty of Psychology, University of eCampus, Novedrate, Italy

6 Virtual Reality Medical Center, La Jolla, CA, USA

7 Virtual Reality Medical Institute, Brussels, Belgium

${ }^{*}$ Corresponding Author: Silvia Serino 


\section{A Free Weekly Self-Administered at-Home Virtual Reality based Protocol for Overcoming the Psychological Burden of \\ Coronavirus: A Pragmatic Waitlist-Controlled Trial Conducted \\ During COVID-19 lockdown in Italy with 2-week Follow-up}

Background: The COVID-19 pandemic is generating extreme psychological stress associated with sadness, grief, irritability, and mood swings. Virtual reality (VR) is an immersive technology that may enhance individuals' personal efficacy, emotional response, and wellbeing.

Objective: We aimed to conduct a single-cohort, controlled trial with a 2:1 (waiting-list - VR protocol) allocation for a selfadministered at-home weekly VR-based protocol for reducing the psychological burden experienced during the COVID-19 lockdown in Italy.

Methods: A national convenience sample of individuals having experienced at least two months of strict social distancing measures (including the stay-at-home order) experienced the following weekly protocol ("COVID Feel Good", available online here: www.covidfeelgood.com) consisting of two integrated parts: the first part consists of a 10-minute $360^{\circ} \mathrm{VR}$ video entitled "Secret Garden" and the second part includes a series of social exercises, with a specific goal for each day of the week. Half of the sample experienced the VR video on the smartphone only, while the other half experienced the VR video using an immersive headset. Primary outcome measures were depressive, anxiety and stress symptoms (measured using the DASS-21, the PSS and the BHS scales). Secondary outcomes were the experienced social connectedness and the level of fear experienced during the COVID-19 pandemic (measured using the SCS and FCOR scales). Analytic methods included different separate linear mixed-effects models.

Results: The study sample was 40 adults [18 females; mean age $30.3 \pm 12.00$ (range: 21-65)]. Results indicated no significant changes $(P=1.000)$ in study variables between the waiting period (i.e., data collected 7 days before the start of the treatment) and the baseline (i.e., data collected at the beginning of the intervention, T0). Participants exhibited improvement from baseline to postintervention for almost all primary variables (all Ps $<0.05$ ), except for the perceived hopelessness $(P=0.1098)$. Results for the secondary outcomes indicated an increase in social connectedness from T0 to T1 ( $P=0.0327)$, but not a significant reduction in the perceived fear of Coronavirus $(P=0.4121)$. Results indicated that treatment positive effects were stable at the 2-weels of follow-up for all study variables, except for depressive 
symptoms. None of the models were significant on the factor "Immersion", suggesting that there is no significant effect of the modality (immersive vs non immersive) on the results of the treatment.

Conclusions: This pragmatic pilot trial tried to understand if and how a weekly VR self-help protocol can help individuals in overcoming the psychological burden associated with spread of the COVID-19 and the consequent health emergency. The results, even if preliminary, suggest the efficacy of the proposed protocol. Moreover, its current availability in 12 different languages - English, Spanish, French, Brazilian/Portuguese, German, Italian, Turkish Japanese, Korean, Farsi, Romanian and Catalan - make COVID Feel Good a free and effective choice for helping individuals worldwide to cope with the psychological distress related to the COVID-19 crisis. 


\section{Introduction}

In late 2019 the virus responsible for the severe acute respiratory syndrome coronavirus 2 COVID-19 was first identified in a small Chinese town ${ }^{1,2}$ forcing the world to face unprecedented medical, economic, and social challenges. After an uncontrollable diffusion of the disease from China to other countries, the World Health Organization (WHO) declared the COVID-19 outbreak a "Public Health Emergency of International Concern" on January $30^{\text {th }}$, 2020. The immense spread of the COVID-19 and the consequent health emergency raised by the growing number of reported cases worldwide led the WHO to declare it a "pandemic" on March 11 th, 20203.

During the first three months of the emergency, Italy was one the most affected countries ${ }^{4}$. The first cases were confirmed between the $21^{\text {st }}$ and the $24 \mathrm{t}^{\mathrm{h}}$ of February 2020 in two areas in the northern regions of Italy, namely the Lombardy and Veneto regions. Two "red zones" were quickly introduced to isolate those areas, introducing physical/social distancing measures to slow down to spread of the disease. On the $8^{\text {th }}$ of March, the Italian government extended these measures to all Lombardy and then to the whole country (the so-called "FaseUno"). Maintaining a social distance of at least 2 meters away from other individuals and limiting social interactions became the first rules to contain the transmission of the virus. Schools and all non-essential business were closed, public events and social meetings were prohibited, working from home became mandatory, where possible. The stay-at-home order (also called "lockdown") resulted in a mass isolation measure that confined individuals to their home place except for essential needs. ${ }^{5,6}$ These measures were implemented worldwide with varying degrees aiming to limit the devasting diffusion of this disease and were partially removed in Italy on the $4^{\text {th }}$ of May when the so-called "Fase-Due" began.

These measures globally achieved their goals of containing the dramatic spread of disease, however they also lead to important psychological consequences and degradation of normal social systems ${ }^{7-11}$. Recent reviews indicated that the short-term psychological consequences included post-traumatic and depressive symptoms, high perceived stress, and an increase in anxiety and sleep disorders. ${ }^{6,12-15}$ A study carried out during the first three weeks of the lockdown in Italy indicated that $37 \%$ of participants experienced post-traumatic stress, while $17-23 \%$ reported significant levels of anxiety, perceived stress, and adjustment disorders ${ }^{16}$. Some authors underlined that such effects can be pervasive and long-lasting, suggesting that the exposure to the psychological stress experienced during the "quarantine" and the consequent social isolation can provoke long-term effects for mental health ${ }^{17,18}$ As reported by Gruber et al. ${ }^{19}$, there are three peculiar and unique ways in which the COVID-19 pandemic is a 
stressor able to adversely affect mental health in global population. First, the pandemic is accompanied by feelings of uncertainty regarding its duration that resulted in increased and diffuse concerns about its long-term economic and social consequences $^{20-22}$. Second, the COVID-19 pandemic is a multidimensional stressor, affecting at the same time multiple spheres of our lives: work, family, and social life. The lockdown period forced families to try to maintain a functional workfamily balance meeting the unique demand of the new working-from-home environment merged with homeschooling ${ }^{23}$. Spinelli and colleagues ${ }^{24}$ investigated the psychological impact of different risk factors associated with the COVID-19 lockdown experience (namely, home environment conditions, individual perceived stress, parent-child dyadic stress) on parents' and children's well-being. They found that the individual perception of lockdown-related difficulties was a crucial factor for the well-being of both parents and children. Third, social distancing measures adopted worldwide had and have enormous costs on the social processes that underlie the very fabric of our ${ }^{25,26}$ On one side, these measures can negatively impact mental health in terms of increase of perceived social isolation, personal distress $8,25,27$ and unhealthy behaviors (e.g., excessive substance use, see for example ${ }^{28}$ ). Besides, adhering to stay-at-home orders and adopting social distancing measures drastically reduce the possibility to access important protective factors against stress, such as an active participation in meaningful social relationships and interactions ${ }^{29,30}$.

Delivering evidence-based psychological interventions for both preventing and treating these mental health disorders is an urgent challenge, considering also that the social distancing measures may limit the adoption of traditional psychological services ${ }^{7,31}$. To facilitate the access of psychological services, traditional psychological interventions has been recently explored and reconfigured as online or telehealth formats ${ }^{31}$. Literature suggests the efficacy of telehealth for delivering psychological interventions ${ }^{32-35}$, and recent studies demonstrated the feasibility of such approaches during the COVID-19 crisis for both health care professionals and the general population ${ }^{36-39}$.

In recent years, there has been a huge increment in the use of technology for the implementation of validated psychological interventions for a wide variety of psychological problems ${ }^{34,40-42}$. Digital psychological self-help interventions can be accessed from any location, with an obvious decrease in logistic and geographical barriers to the treatment delivery, they are often more affordable and less time-consuming in comparison to traditional interventions, with a subsequent lower impact on the public health service, and they significantly improve self-monitoring processes. ${ }^{43-47,49}$ A recent meta-analysis reviewed 34 studies with different type of self-guided therapeutic approaches (including cognitive-behavioral, mindfulness, and acceptance-based interventions) and 
globally these approaches showed small to medium effects in comparison to control groups ${ }^{48}$. In particular, advanced technologies, such as Virtual Reality (VR) could play a unique role in times of COVID-19 pandemic by supporting the delivery of accessible and affordable self-guided therapeutic interventions that offer multiple advantages, considering also high-treatment costs and the limited availability of health professionals ${ }^{31,50,51}$

The present controlled trial tested the effectiveness of COVID Feel Good $^{52}$, a novel self-help VR-based protocol aimed at helping individuals in cope with the psychological burden related to COVID-19 pandemic and restrictive social distancing measures. The protocol is currently freely available online (http://www.covidfeelgood.com) in 12 different languages - English, Spanish, French, Brazilian/Portuguese, German, Italian, Turkish Japanese, Korean, Farsi, Romanian and Catalan. The protocol consists of watching a 10-minute three hundred sixty-degree $\left(360^{\circ}\right)$ VR video, titled the "The Secret Garden," at least once a day for a week. Each day, after the VR protocol, participants were invited to perform series of social exercises with targeted goals for each day of the week. To guarantee scalability, the protocol could be accessed both through participants' smartphone and basic low-cost cardboard VR headset (immersive modality) or through the YouTube App (non-immersive modality).

We hypothesized that the weekly VR-based self-help protocol COVID Feel Good would be associated with a reduction of depressive and anxiety symptoms, general distress, perceived levels of stress, hopelessness (primary outcome measures), with an increase in interpersonal closeness with the social world and a decrease of fear of COVID-19 (secondary outcome measures) at the end of treatment compared with responses at 1-week waiting period and baseline responses. We predicted also that treatment gains would be maintained at 2weeks follow-up. Additionally, we also evaluated the pre-post changes in perceived levels of relaxation and distress by comparing responses collected each day during the 1 -week waiting with those collected after completing each daily treatment module.

\section{Methods}

\section{1 Study design}

The present study was a pragmatic trial ${ }^{53}$ aimed at investigating the efficacy of COVID Feel Good protocol implemented in daily life routine during the COVID-19 pandemic. We employed a single-cohort, controlled trial with a 2:1 (waiting-list - VR protocol) allocation and we compared changes in levels of depression, anxiety, general distress, perceived stress, and hopelessness, social connectedness, and fear of COVID-19 between a control waiting period (i.e., 7 days before the start of the intervention - Waiting Period), changes before the start of the treatment (Day $0-\mathrm{T} 0$ ) and the end of the treatment period (Day $7-\mathrm{T} 1$ ). 
The inclusion of a 2-week follow-up (Day 21 - T2) allowed us to additionally evaluate the stability of the treatment effects. State measures of anxiety, perceived stress, mood feelings and relaxation levels were collected daily in the waiting period ( 7 days before the start of the intervention) and after the completion of each treatment module, from Day 0 to Day 7 . The study was conducted in accordance with the principles of the 1964 Declaration of Helsinki and approved by the ethical committee of the Istituto Auxologico Italiano, IRCCS. All participants gave informed consent prior to participating by email.

\subsection{Procedure and sample}

From June and July 2020, participants were recruited through combined use of traditional strategies and advertisement on social media platform in Italy. Potential participants were directly contacted by one of the researchers through email and asked to complete a brief online questionnaire for screening purposes. Inclusion criteria were being at least 18 years old, being resident in Italy, having experienced at least two months of strict social distancing measures (including stay-at-home order) implemented by the Italian National Government (precise information about the measures implemented can be found here ${ }^{54}$ ), availability and agreement of a partner for conducting the relational component of the treatment, availability of a smartphone with Internet access, and normal or corrected-to-normal vision. Exclusion criteria (all self-reported) included the presence of a diagnosis of major mental disorder and lack of stereoscopic vision or a balance/vestibular problem that would disrupt the VR experience. Participants who met the above-mentioned eligibility criteria were provided with information about study design and asked to sign the informed consent. Considering an anticipated effect size (f) of 0.25 , we estimated a sample size of 36 participants, with a desired power of $0.80(1-\beta)$ on between and within-group comparisons, via G*Power 3.1 software. Accordingly, a total of 40 participants [ 18 females; mean age $30.3 \pm 12.00$ (range: $21-65$ )] were enrolled in the study.

Table 1 presents the sample demographic characteristics. After the inclusion, they received the psychological assessment survey including all the outcome measures to be completed online through a Qualtrics survey and they were informed that their intervention would start after a 1-week waiting period. When the waiting period was over, participants received another Qualtrics survey to complete (the T0 baseline psychological assessment) with instructions to start the protocol and the self-help materials as an email attachment. During the treatment week, each day they received an email with the state assessment battery through a Qualtrics link to be completed at the end of each VR treatment module. At the end of the week (Day 7 - T1) and after 2-week follow-up (Day 21 
- T2), participants were invited to fill again the assessment survey with the same outcome measures as in the baseline.

\subsection{The protocol}

Participants received six modules consisting of two integrated parts: the first part consists of a 10 -minute $360^{\circ}$ VR video entitled "Secret Garden" and the second part includes a series of social exercises, with a specific goal for each day of the week. From a technological viewpoint, videos $360^{\circ}$ are spherical videos recorded with omnidirectional cameras to collect images from all around the environment. $360^{\circ}$ videos can be captured in natural landscapes or created using $3 \mathrm{D}$ computer graphics software $\left(360^{\circ} \mathrm{VR}\right.$ videos). They can be displayed in immersive (i.e., through a Head Mounted Display or low-cost cardboard connected with a smartphone) or in a non-immersive way (i.e., for instance, YouTube supports $360^{\circ}$ video formats both in its Android app and website). During the playback, users can actively move the camera and have a $360^{\circ}$ view of the environment. This feature allows them to have multiple views of the same video content interactively, feeling inside the represented environment and being the protagonist of the action that unfolds in front of their eyes.

The $360^{\circ}$ VR video "The Secret Garden" (Figure 1) has been developed through an iterative process involving psychologists, 3D artists, musicians, storytellers, and designers. It has been validated in previous studies by Chirico and colleagues ${ }^{55}$. It immerses users in a beautiful and relaxing Japanese garden, providing them with the visual (i.e., the flow of water) and auditory (i.e., the sound of running water) elements available in natural environments. The virtual environment has been developed using the software Unreal Engine. The immersive experience is accompanied by a relaxation induction narrative structured following the principles of Compassion Focused Therapy56,57. This relaxation technique aims at deactivating the human threat protection system and activating the soothing system, with a mindset devoted to giving and receiving care, affecting, and nurturance. At the end of the VR exposure, participants were invited to perform a series of social task related to personal identity and interpersonal relationships. These tasks have been developed following guidelines provided by Winch ${ }^{58}$ to cope with emotional experiences that can generate emotional pain, loneliness, rejection, or rumination. The tasks have the following general aims: a) helping participants to pay attention and recognize their emotional discomfort; b) supporting participants to reinforce their coping skills; c) helping participants to monitor themselves and protect self-esteem; d) support participants in finding a personal meaning even in difficult times. In addition, each activity has a specific objective: 
1. Day 1: Support to cope with stress, negative intrusive thoughts, worries related to the COVID-19 pandemic;

2. Day 2: Increase of self-esteem;

3. Day 3: Promote the use of autobiographical memories to create a stable representation of themselves over time, as well as increase intimacy and connectedness by sharing personal memories;

4. Day 4: Enhance of sense of community

5. Day 5: Promote conscious self-regulation and self-organization of life goals;

6. Day 6: Increase of empathy;

7. Day 7: Support a long-term psychological change.

All the exercises are designed to be experienced with another person (not necessarily physically together), to facilitate a process of critical examination and eventual revision of core assumptions and beliefs related to personal identity, relationships, and goals. The full description of the sessions is provided in 52 .

\subsection{Outcome measures}

The following self-report questionnaires were included in the psychological assessment survey filled online through a Qualtrics survey and they have been administered at the different time intervals to investigate changes in the subjective experience during a control waiting period ( 7 days before the start of the intervention - Waiting Period), before (Day $0-\mathrm{T} 0$ ) and at the end of the treatment period (Day 7 - T1), and at 2-weeks later follow-up (Day 21 - T2).

\subsubsection{Primary outcome measure}

- Depression Anxiety Stress Scale (DASS-21)59,60: The DASS-21 is the short version of the original self-report questionnaire developed and validated by Lovibond et al. ${ }^{59}$ to evaluate depressive, anxiety and stress symptoms. It is composed of 21 items, with 7 items per subscale: depression, anxiety, and stress. Participants are asked to score every item on a scale from 0 ("did not apply to me at all") to 3 ("applied to me very much"). Sum scores are computed for the three subscales, but it is also possible to compute a composite score of "general distress" resulting from the sum of all items. Accordingly, total scale may range between 0 and 63 and subscales may range between 0 and 21 .

- Perceived Stress Scale (PSS) ${ }^{61,62}$ : The PSS is a self-report questionnaire for evaluating individuals' perceived stress. The scale is composed of 10 items on a 5-point Likert, and it measures to which extent our daily experiences are subjectively perceived as stressful in the last month. The current study, it has been 
adapted to evaluate the perceived level of stress in the last week. It yields a composite score of perceived stress resulting from the sum of the responses of single items, from 0 to 40 .

- Beck Hopelessness Scale (BHS) ${ }^{63,64}$ : The BHS is a self-report questionnaire for measuring pessimistic thoughts or negative attitude towards the future in three different life spheres: feeling about the future, loss of motivation, and general expectations. It is composed of 20 true-false items. The total sum score can range from 1 to 20, with higher scores underscore higher levels of hopelessness.

\subsubsection{Secondary outcome measures}

- Social Connectedness Scale (SCS)65,66: The SCS (42) is a self-report instrument that measures the extent to which the individual feels connected to other persons or the social context. The scale is composed of 8 items on a 6-point Likert scale. Sum scores can range from 0 to 48 , with higher scores indicate a higher sense of social connectedness.

- Fear of Coronavirus (FCOR) 67,68: FCOR is a scale aimed to measure the level of fear experienced during the COVID-19 pandemic ("I am most afraid of coronavirus-19"). FCOR is composed of 7 items on a 5-point. Sum scores can range from 0 to 35 , with higher scores indicate a higher fear of COVID-19.

\subsubsection{State outcome measures}

Furthermore, the following self-report questionnaires were administered each day to measure changes in daily subjective experience from the waiting period ( 7 days before the starting of the protocol) and after the completion of each daily session (from Day 0 to Day 7):

- State-Trait Anxiety Inventory (STAI) ${ }^{69,70:}$ The STAI is a 40 -item self-report questionnaire on a 4-points Likert scale where participants are asked to evaluate both state and trait levels of anxiety. This questionnaire is divided into 20 items that refer to the state anxiety (STAI-S), and 20 items that refer to the trait anxiety (STAI-T). In this study, we used only the STAI-S. Total scores range from 20 to 80, where higher scores indicate higher anxiety feelings.

- Smith Relaxation State Inventory 3 (SRSI3) ${ }^{71}$ : The SRSI3 is the revised version of the Smith Relaxation State inventory and it measures 19 relaxation states. Individuals are asked to evaluate how they feel right now in response to 38 items on a 6-point Likert scale. The scale is divided into four subscales that can be selected independently. For this protocol, we selected the following subscales: or this protocol, the following subscales have been selected, for a total of 20 items: 
rest/refresh, energized, physical relaxation, at ease/peace, joy, mental quiet, awareness, somatic stress, emotional stress, and cognitive stress.

- Subjective Units of Distress Scale (SUDS) ${ }^{72}$ : The SUDS is a numeric scale rating scale from 0 to 100 that evaluates the subjectively perceived level of distress.

\subsection{Data analysis}

Linear mixed-effects models (LMMs, estimated using REML and nloptwrap optimizer) were fitted to evaluate the effectiveness of the treatment. These models estimate both inter-individual variability and intra-individual patterns of change over repeated sessions, thus controlling the issue of non-independence among repeated measures. ${ }^{73}$ Two separate models were fitted to evaluate changes for each outcome score (DASS-21_Depression, DASS-21_Anxiety, DASS21_Stress, DASS-21_General Distress, PSS, BHS, SCS, FCOR) across four-time points from the waiting period (7 days before the start of the treatment - Waiting) to the follow-up phase (T2). The first model was run according to the following formula $=$ Outcome $\sim$ Time, and the second one according to this formula $=$ Outcome $\sim$ Time ${ }^{*}$ Immersion, to check for the potential effect of using immersive vs. non-immersive modality on the results. All models included participants as a random effect (formula $=\sim 1 \mid$ ID). Table S1 (Supplementary Information) presents the parameters estimated for each model that was fitted. In model comparisons, we selected the most parsimonious model according to Akaike's Information Criteria (AIC) and Bayesian Information Criteria, (BIC) to evaluate which model resulted to better fit the data.

Then, we applied the same pipeline for evaluating changes in the daily subjective experience (STAI-S, SRSI3- Rest/Refresh, SRSI3energized, SRSI3Physical relaxation, SRSI3- at ease/peace, SRSI3- Joy, SRSI3- Mental quiet, SRSI3- Aware, SRSI3- Somatic stress, SRSI3- Emotional stress, SRSI3- cognitive stress, and SUDS) between the waiting period and the treatment week. Daily measures were first averaged to obtain a measure of global subjective experience before the starting of the treatment (waiting list period) and after the treatment sessions (treatment period). Table S2 (Supplementary Information) shows the parameters estimated for each model that was fitted. Analyses of variance (ANOVA) on selected LMM parameters were performed with Kenward-Roger approximation for degrees of freedom ${ }^{74}$. Significant effects were examined with post-hoc comparisons (Bonferroni's adjustment) and were performed with emmeans and are reported with estimated marginal means (EEM) and standard error (SE). Assumptions for all models were satisfied. The Linear Mixed Models were run in R Studio Version 1.1.463 with the following packages Lme4 ${ }^{75}$ with restricted maximum likelihood ${ }^{76}$, ImerTest ${ }^{77}$, emmeans ${ }^{78}$. 


\section{Results}

\subsection{Primary and secondary outcome measures}

Table 2 displays the means for primary and secondary outcomes across each assessment. None of the models were significant on the factor "immersion", suggesting that there is no significant effect of the modality (immersive vs non immersive) on the results of the treatment. Furthermore, the models that include the factor immersion reported higher values of AIC and BIC parameters, suggesting that the models according to this formula: = Outcome $\sim$ Time, were the best fit for the data. Analysis of variance (ANOVA) on the LMM's parameters indicated a significant effect of Time for the primary outcomes of depression $[F(3$, $115.2)=6.68098 ; P<0.001]$, stress $[F(3,115.17)=6.3453 ; P<0.001]$, general distress $[F(3,115.15)=6.9713 ; P<0.001]$, perceived stress $F(3,115.17)=5.1457$; $P=0.002]$, and hopelessness $[F(3,115.07)=4.7977, P=0.003)]$. Among the secondary outcomes, ANOVA on the Linear Mixed Model's parameters indicated a significant effect of Time on social connectedness $[F(3,115.15)=5.4757$, $P=0.001)]$ and fear of coronavirus $[F(3,115.07)=5.0214, P=0.003)]$. No significant effect of Time was found on anxiety, as measured by DASS-21 $[F(3,115.2)=$ 2.2556, $P>0.05)]$. These significant effects were followed up with post-hoc (Bonferroni adjusted) comparisons testing to determine changes in outcomes across the different time points. Full comparisons are presented in Table 3.

Results indicated no significant changes $(P=1.000)$ in all study variables between the waiting period (i.e., data collected 7 days before the start of the treatment) and the baseline (i.e., data collected at the beginning of the intervention, T0). Participants exhibited improvement from baseline to postintervention for all primary variables (all $\mathrm{Ps}<0.05$ ), except for the perceived hopelessness $(P=0.1098)$. Results for the secondary outcomes indicated, an increase in social connectedness from T0 to $\mathrm{T} 1 \quad(\mathrm{P}=0.0327)$, but not a significant reduction in perceived fear of Coronavirus ( $\mathrm{P}=0.4121)$.

Among all the study variables, these significant improvements were maintained from postintervention to the 2-weeks follow-up $(P>0.05)$, except for an observed increase in depressive symptoms $(P=0.005)$.

\subsection{State outcome measures}

None of the models were significant on the factor "immersion", suggesting that there is no significant effect of type of modality (immersive vs non immersive) on the results of the treatment. Furthermore, the models that include the factor immersion reported higher values of $\mathrm{AIC}$ and BIC parameters, suggesting that the models according to this formula: $=$ Outcome $\sim$ Time, were the best fit for the data. As concerns, the model included the subscale SRSI3- Aware, results indicated that no effect of the neither of the Time or Immersion (see Table S2). 
Analysis of variance (ANOVA) on the LMM's parameters indicated a significant of Time for anxiety levels $[F(1,39.323)=39.993 ; P<0.001]$, refreshed feelings $[F(1$, 39.732 $)=6.3453 ; P=0.007]$, energized feelings $[F(1,39.836)=5.54 ; P=0.023]$, perceived physical relaxation $F(1,39.634)=25.746 ; P<0.001]$, feelings of peace $[F(1,40.242)=5.2272, P=0.027)]$, feelings of mental quiet $[F(1,39.558)=31.363$, $P<0.001)]$, perceived somatic distress $[F(1,39.156)=31.594, P<0.001)]$, perceived emotional distress $[F(1,39.348)=15.356, P<0.001)]$, perceived cognitive distress $[F(1,39.178)=31.508, P<0.001)]$, and subjectively perceived levels of discomfort $[F(1,39.206)=25.585, P<0.001)]$. No significant of Time emerged for perceived feelings of joy $[F(1,39.285)=3.8287, P=0.057)]$. Table 4 displays the means of the state outcome measures across the time points. An improvement in almost all study variables was observed from the waiting period (i.e., data collected each day, for 7 days before the start of the intervention) to the intervention phase, namely data collected each day after each treatment module.

\section{Discussion}

The aim of this study was to evaluate the effectiveness of a novel Virtual Reality (VR) self-help psychological protocol to helping individuals coping with the psychological burden related to the COVID-19 pandemic and its related social restrictions measures. The entire protocol ( 1 week) was based on the $360^{\circ}$ video (VR) "Secret Garden", which simulates a natural environment aiming to promote relaxation and self-reflection. The "Secret Garden" experience was combined with daily social exercises with targeted goals that are designed to be experienced with another person. The sample consisted of Italian participants who experienced at least two months of strict social distancing measures, including a "stay-at-home" order, implemented by the Italian National Government to contain the dramatic spread of the disease in the country.

First, results showed no significant changes in all outcome measures between the waiting period (i.e., 7 days before the start of the protocol) and the start of the intervention. At the end of the intervention, participants experienced improvements in depressive feelings and perceived stress levels (primary outcomes), and in their perceived connection to the social world (secondary outcome).

We did not observe any effect of the treatment as concerns and the subjective perception of hopelessness. An explanation might be offered by Cipolletta and Ortu ${ }^{79}$, who suggested that one of the most profound psychological effects of the COVID-19 crisis is that this period has suspended time and our future ${ }^{79}$, with a significant rebound in feelings of hopelessness and demoralization 19. results also indicated the absence of significant effect of treatment on anxiety. Results indicated that treatment positive effects were stable at the 2-weels of follow-up for these study variables, except for depressive symptoms. A slight 
increase in depression levels was indeed observed from the end of the treatment to the follow-up assessment period. These results could be explained with data reviewed by recent meta-analyses indicating that the prevalence of depressive symptoms among the general population appears to be 3-fold higher during the COVID-19 pandemic ${ }^{80}$ or even 7 -fold higher as compared to $2017^{81}$. In particular, a study ${ }^{82}$ carried out in Italy between March and May 2020 on 20,720 participants found that $2.4 \%$ of participants reported severe or extremely severe levels of depressive symptoms and $17.6 \%$ participants exhibited high levels of anxiety, as measured on DASS-21 questionnaire. This result might also explain why we did not find a significant effect of the intervention on anxiety feelings. Although a huge decrease in anxiety feelings from the baseline to postintervention was observed (T0 mean: $5.15 ; \mathrm{SD}=4.77$ vs. $\mathrm{T} 1$ mean: $3.45 \mathrm{SD}=4.51$ ), we also measured a slight increase in anxiety levels from the waiting period to the start of the protocol.

Besides, an improvement was observed regarding the daily levels of anxiety, perceived stress, and mood feelings from the waiting period to the treatment week, further suggesting the efficacy treatment in ameliorating participants' subjective daily state mood and experience. Participants had the opportunity to follow the protocol with the use of a head-mounted display (HMD, immersive modality) or without it, namely by watching the $360^{\circ}$ video Secret Garden on Youtube (nonimmersive modality). We did not find any difference in all outcome measures as concerns the immersion, suggesting that the intervention effectiveness was transtechnological and, therefore, able to reach a larger population, which has no direct access to advanced technological device, such as the use of the HMD.

Given the importance of delivering and testing digital psychological treatments to help individuals cope with psychological distress related to the COVID-19 crisis ${ }^{10,39,83}$, the results obtained might have important implications for future evidence-based protocols in this field. As explained in the Introduction, VR occupies a prominent place among all other technologies for delivering self-help protocol for several reasons ${ }^{41,42,84}$. It is traditionally employed as a "simulative instrument" 41 to reproduce ecologically valid and interactive scenarios recreating feared/critical situations (i.e., fear of spider), while allowing the anxiety to attenuate, or naturalistic situations where they can learn specific skills in a safe, ecological, and interactive environment. At the same time, VR-based protocols allow precise control over the sessions' delivery, according to both to therapeutic strategies and patients' progress; this aspect is particularly relevant for self-help protocol administered in absence of the therapists' guidance. These advantages offered by VR has been already exploited in VR-based self-help treatments for treating phobias and anxiety disorders ${ }^{85,8643}$ Here, we have further exploited the potentiality of VR as a "simulative instrument" to provide participants with the opportunity to be immersed in a naturalistic and safe digital place ${ }^{55}$, far from the 
stressful situations experienced in routine daily contexts, where they can learn how to relax and reflect upon their experience following a guided protocol. This effect has been enhanced by creating a bridge with the real-life context with different daily social tasks aiming at facilitating a process of critical examination and eventually revision of core assumptions and beliefs ${ }^{52}$.

\subsection{Limitations}

This study has several limitations. First, as noted earlier, although our study was not aimed at comparing the use of immersive vs. non-immersive in VR-based selfhelp treatments, we did not experimentally control this variable. Future research could aim to specifically examine the impact of immersion in the delivery of digital psychological self-help treatments. Second, given the current context, we opted to not include a control group but to offer to all participants the possibility to participate in the study. The absence of a proper control condition may have impacted the results. Future studies should further investigate the efficacy of COVID-19 Feel Good in comparison to standard psychological treatments. Third, the participation in the protocol was limited to individuals with proper access to a smartphone/pc with Internet access. This might have impacted the generalizability of the results obtained.

\subsection{Conclusion}

The psychological and social costs of the COVID-19 pandemic are now evident. If do not properly and early treated with appropriate evidence-based interventions, this psychological burden of COVID-19 might have a long-term health effect ${ }^{7,8}$. Globally, this study has important implications for the design and delivery of digital self-help psychological protocol to alleviate the psychological distress associated with the pandemic. This pragmatic pilot trial tried to understand if and how a weekly VR self-help protocol can help overcome the psychological burden of the coronavirus. The results, even if preliminary, suggest the efficacy of the proposed protocol. More, its current availability in 12 different languages - English, Spanish, French, Brazilian/Portuguese, German, Italian, Turkish Japanese, Korean, Farsi, Romanian and Catalan - make COVID Feel Good an effective choice for helping individuals worldwide to cope with psychological distress related to the COVID-19 crisis. However, future works are needed to evaluate the efficacy of this protocol in other groups (i.e., health professionals, or older adults), other countries and its impact in the longer term. 


\section{References}

1. Paules, C.I., Marston, H.D., and Fauci, A.S. (2020). Coronavirus Infections-More Than Just the Common Cold. JAMA 323, 707-708.

2. Roser, M., Ritchie, H., Ortiz-Ospina, E., and Hasell, J. (2020). Coronavirus pandemic (COVID-19). Our world data.

3. Jee, Y. (2020). WHO international health regulations emergency committee for the COVID-19 outbreak. Epidemiol. Health 42.

4. Remuzzi, A., and Remuzzi, G. (2020). COVID-19 and Italy: what next? Lancet 395, 12251228.

5. Katz, R., Vaught, A., and Simmens, S.J. (2019). Local decision making for implementing social distancing in response to outbreaks. Public Health Rep. 134, 150-154.

6. Brooks, S.K., Webster, R.K., Smith, L.E., Woodland, L., Wessely, S., Greenberg, N., and Rubin, G.J. (2020). The psychological impact of quarantine and how to reduce it: rapid review of the evidence. Lancet 395, 912-920.

7. Holmes, E.A., O'Connor, R.C., Perry, V.H., Tracey, I., Wessely, S., Arseneault, L., Ballard, C., Christensen, H., Silver, R.C., and Everall, I. (2020). Multidisciplinary research priorities for the COVID-19 pandemic: a call for action for mental health science. The Lancet Psychiatry.

8. Vindegaard, N., and Benros, M.E. (2020). COVID-19 pandemic and mental health consequences: Systematic review of the current evidence. Brain. Behav. Immun. 89, 531542.

9. Marazziti, D., Pozza, A., Di Giuseppe, M., and Conversano, C. (2020). The psychosocial impact of COVID-19 pandemic in Italy: A lesson for mental health prevention in the first severely hit European country. Psychol. Trauma Theory, Res. Pract. Policy 12, 531

10. Pfefferbaum, B., and North, C.S. (2020). Mental health and the Covid-19 pandemic. N. Engl. J. Med. 383, 510-512.

11. Pagnini, F., Bonanomi, A., Tagliabue, S., Balconi, M., Bertolotti, M., Confalonieri, E., Di Dio, C., Gilli, G., Graffigna, G., and Regalia, C. (2020). Knowledge, concerns, and behaviors of individuals during the first week of the coronavirus disease 2019 pandemic in Italy. JAMA Netw. open 3, e2015821-e2015821.

12. De Giorgio, A. (2020). Global psychological implications of Severe Acute respiratory syndrome Coronavirus 2 (SARS-CoV-2) and Coronavirus disease-2019 (COVID-19). What can be learned from Italy. Reflections, perspectives, opportunities. Front. Psychol. 11.

13. Xiong, J., Lipsitz, O., Nasri, F., Lui, L.M.W., Gill, H., Phan, L., Chen-Li, D., lacobucci, M. Ho, R., and Majeed, A. (2020). Impact of COVID-19 pandemic on mental health in the general population: A systematic review. J. Affect. Disord.

14. Franceschini, C., Musetti, A., Zenesini, C., Palagini, L., Scarpelli, S., Quattropani, M.C., Lenzo, V., Freda, M.F., Lemmo, D., and Vegni, E. (2020). Poor sleep quality and its consequences on mental health during the COVID-19 lockdown in Italy. Front. Psychol. 11, 3072.

15. Rubin, G.J., and Wessely, S. (2020). The psychological effects of quarantining a city. Bmj 368.

16. Rossi, R., Socci, V., Talevi, D., Mensi, S., Niolu, C., Pacitti, F., Di Marco, A., Rossi, A., Siracusano, A., and Di Lorenzo, G. (2020). COVID-19 pandemic and lockdown measures impact on mental health among the general population in Italy. Front. psychiatry 11, 790.

17. Park, S.-C., and Park, Y.C. (2020). Secondary emotional reactions to the COVID-19 outbreak should be identified and treated in Korea. J. Korean Med. Sci. 35.

18. Fernández, R.S., Crivelli, L., Guimet, N.M., Allegri, R.F., and Pedreira, M.E. (2020). Psychological distress associated with COVID-19 quarantine: Latent profile analysis, outcome prediction and mediation analysis. J. Affect. Disord. 277, 75-84.

19. Gruber, J., Prinstein, M.J., Clark, L.A., Rottenberg, J., Abramowitz, J.S., Albano, A.M., Aldao, A., Borelli, J.L., Chung, T., and Davila, J. (2020). Mental health and clinical psychological science in the time of COVID-19: Challenges, opportunities, and a call to action. Am. Psychol.

20. Ruffolo, M., Price, D., Schoultz, M., Leung, J., Bonsaksen, T., Thygesen, H., and Geirdal, A.Ø. (2021). Employment uncertainty and mental health during the COVID-19 pandemic initial social distancing implementation: A cross-national study. Glob. Soc. Welf., 1-10.

21. Rettie, H., and Daniels, J. (2020). Coping and tolerance of uncertainty: Predictors and mediators of mental health during the COVID-19 pandemic. Am. Psychol.

22. Satici, B., Saricali, M., Satici, S.A., and Griffiths, M.D. (2020). Intolerance of uncertainty and mental wellbeing: serial mediation by rumination and fear of COVID-19. Int. J. Ment. Health Addict., 1-12.

23. Thorell, L.B., Skoglund, C., de la Peña, A.G., Baeyens, D., Fuermaier, A.B.M., Groom, M.J., Mammarella, I.C., van der Oord, S., van den Hoofdakker, B.J., Luman, M., et al. (2021). Parental experiences of homeschooling during the COVID-19 pandemic: differences between seven European countries and between children with and without mental health conditions. Eur. Child Adolesc. Psychiatry.

24. Spinelli, M., Lionetti, F., Pastore, M., and Fasolo, M. (2020). Parents' stress and children's psychological problems in families facing the COVID-19 outbreak in Italy. Front. Psychol. 11,1713

25. Townsend, A.K., Hawley, D.M., Stephenson, J.F., and Williams, K.E.G. (2020). Emerging infectious disease and the challenges of social distancing in human and non-human animals. Proc. R. Soc. B 287, 20201039. 
26. Riva, G., Wiederhold, B.K., and Mantovani, F. (2021). Surviving COVID-19: The Neuroscience of Smart Working and Distance Learning. Cyberpsychology, Behav. Soc. Netw. 24, 79-85.

27. Killgore, W.D.S., Cloonan, S.A., Taylor, E.C., and Dailey, N.S. (2020). Loneliness: A signature mental health concern in the era of COVID-19. Psychiatry Res. 290, 113117

28. Rodriguez, L.M., Litt, D.M., and Stewart, S.H. (2020). Drinking to cope with the pandemic: The unique associations of COVID-19-related perceived threat and psychological distress to drinking behaviors in American men and women. Addict. Behav. 110, 106532.

29. Magson, N.R., Freeman, J.Y.A., Rapee, R.M., Richardson, C.E., Oar, E.L., and Fardouly, J. (2021). Risk and protective factors for prospective changes in adolescent mental health during the COVID-19 pandemic. J. Youth Adolesc. 50, 44-57.

30. Ye, Z., Yang, X., Zeng, C., Wang, Y., Shen, Z., Li, X., and Lin, D. (2020). Resilience, social support, and coping as mediators between COVID-19-related stressful experiences and acute stress disorder among college students in China. Appl. Psychol. Heal. WellBeing 12, 1074-1094.

31. Zhou, X., Snoswell, C.L., Harding, L.E., Bambling, M., Edirippulige, S., Bai, X., and Smith, A.C. (2020). The role of telehealth in reducing the mental health burden from COVID-19. Telemed e-Health 26, 377-379.

32. Luxton, D.D., Pruitt, L.D., and Osenbach, J.E. (2014). Best practices for remote psychological assessment via telehealth technologies. Prof. Psychol. Res. Pract. 45, 27.

33. Hughes, C., and Ebadat, D. (2017). Upper extremity injuries in sub-Saharan Africa. In 2017 IST-Africa Week Conference, IST-Africa 2017 (Institute of Electrical and Electronics Engineers Inc.).

34. Riva, G. (2000). From Telehealth to E-health: Internet and distributed virtual reality in health care. CyberPsychology Behav. 3, 989-998.

35. Langarizadeh, M., Tabatabaei, M.S., Tavakol, K., Naghipour, M., Rostami, A., and Moghbeli, F. (2017). Telemental health care, an effective alternative to conventional mental care: a systematic review. Acta Inform. Medica 25, 240.

36. Connolly, S.L., Stolzmann, K.L., Heyworth, L., Weaver, K.R., Bauer, M.S., and Miller, C.J. (2020). Rapid increase in telemental health within the Department of Veterans Affairs during the COVID-19 pandemic. Telemed. e-Health.

37. Madigan, S., Racine, N., Cooke, J.E., and Korczak, D.J. (2020). COVID-19 and telemental health: Benefits, challenges, and future directions. Can. Psychol. Can.

38. Wright, J.H., and Caudill, R. (2020). Remote treatment delivery in response to the COVID19 pandemic. Psychother. Psychosom. 89, 1.

39. Mishkind, M.C., Shore, J.H., Bishop, K., D'Amato, K., Brame, A., Thomas, M., and Schneck, C.D. (2020). Rapid Conversion to Telemental Health Services in Response to COVID-19: Experiences of Two Outpatient Mental Health Clinics. Telemed. e-Health.

40. Luxton, D.D., McCann, R.A., Bush, N.E., Mishkind, M.C., and Reger, G.M. (2011). mHealth for mental health: Integrating smartphone technology in behavioral healthcare Prof. Psychol. Res. Pract. 42, 505.

41. Riva, G., Wiederhold, B.K., and Mantovani, F. (2019). Neuroscience of virtual reality: from virtual exposure to embodied medicine. Cyberpsychology, Behav. Soc. Netw. 22, 82-96.

42. Valmaggia, L.R., Latif, L., Kempton, M.J., and Rus-Calafell, M. (2016). Virtual reality in the psychological treatment for mental health problems: An systematic review of recent evidence. Psychiatry Res. 236, 189-195.

43. Villani, D., Grassi, A., Cognetta, C., Toniolo, D., Cipresso, P., and Riva, G. (2013). Selfhelp stress management training through mobile phones: An experience with oncology nurses. Psychol. Serv. 10, 315

44. Beatty, L., and Lambert, S. (2013). A systematic review of internet-based self-help therapeutic interventions to improve distress and disease-control among adults with chronic health conditions. Clin. Psychol. Rev. 33, 609-622.

45. Karyotaki, E., and van Ballegooijen, W. (2020). Digital self-help interventions for suicidal ideation and behaviour. Lancet Digit. Heal. 2, e4-e5.

46. Yim, S.H., and Schmidt, U. (2019). Experiences of computer-based and conventional selfhelp interventions for eating disorders: A systematic review and meta-synthesis of qualitative research. Int. J. Eat. Disord. 52, 1108-1124.

47. Riva, G., and Wiederhold, B.K. (2020). How cyberpsychology and virtual reality can help us to overcome the psychological burden of coronavirus.

48. Fischer, R., Bortolini, T., Karl, J.A., Zilberberg, M., Robinson, K., Rabelo, A., Gemal, L., Wegerhoff, D., Nguyễn, T.B.T., and Irving, B. (2020). Rapid review and meta-metaanalysis of self-guided interventions to address anxiety, depression, and stress during COVID-19 social distancing. Front. Psychol. 11.

49. Riva, G., Mantovani, F., and Wiederhold, B.K. (2020). Positive Technology and COVID19. Cyberpsychology, Behav. Soc. Netw. 23, 581-587.

50. Pincus, L.E. (2020). Telemental health during a global pandemic: Clinical lessons from guided self-help, telephone therapy case studies. Pragmatic Case Stud. Psychother. 16, 118-123.

51. Riva, G., and Riva, E. (2020). COVID Feel Good: A Free VR Self-Help Solution for Providing Stress Management and Social Support During the COVID-19 Pandemic. Cyberpsychology, Behav. Soc. Netw. 23, 652-653.

52. Riva, G., Bernardelli, L., Browning, M.H.E.M., Castelnuovo, G., Cavedoni, S., Chirico, A., Cipresso, P., de Paula, D.M.B., Di Lernia, D., and Fernández-Álvarez, J. (2020). COVID feel good-an easy self-help virtual reality protocol to overcome the psychological burden of coronavirus. Front. Psychiatry $11,996$. 
53. Welsing, P.M., Rengerink, K.O., Collier, S., Eckert, L., van Smeden, M., Ciaglia, A., Nachbaur, G., Trelle, S., Taylor, A.J., and Egger, M. (2017). Series: Pragmatic trials and real world evidence: Paper 6. Outcome measures in the real world. J. Clin. Epidemiol. 90, 99-107.

54. Salute, M. Della .

55. Chirico, A., Clewis, R.R., Yaden, D.B., and Gaggioli, A. (2020). Nature versus Art as Elicitors of the Sublime: A Virtual Reality study.

56. Gilbert, P. (2009). Developing a compassion-focused approach in cognitive behavioural therapy. Cogn. Behav. Ther. A Guid. Pract. Clin. 2, 205-220.

57. Gilbert, P. (2010). An introduction to compassion focused therapy in cognitive behavior therapy. Int. J. Cogn. Ther. 3, 97-112.

58. Winch, G. (2013). Emotional first aid: Practical strategies for treating failure, rejection, guilt, and other everyday psychological injuries (Exisle Publishing).

59. Lovibond, P.F., and Lovibond, S.H. (1995). The structure of negative emotional states: Comparison of the Depression Anxiety Stress Scales (DASS) with the Beck Depression and Anxiety Inventories. Behav. Res. Ther. 33, 335-343.

60. Bottesi, G., Ghisi, M., Altoè, G., Conforti, E., Melli, G., and Sica, C. (2015). The Italian version of the Depression Anxiety Stress Scales-21: Factor structure and psychometric properties on community and clinical samples. Compr. Psychiatry 60, 170-181.

61. Cohen, S., Kamarck, T., and Mermelstein, R. (1994). Perceived stress scale. Meas. Stress A Guid. Heal. Soc. Sci. 10,1-2.

62. Mondo, M., Sechi, C., and Cabras, C. (2019). Psychometric evaluation of three versions of the Italian Perceived Stress Scale. Curr. Psychol., 1-9.

63. Beck, A.T., Weissman, A., Lester, D., and Trexler, L. (1974). The measurement of pessimism: the hopelessness scale. J. Consult. Clin. Psychol. 42, 861

64. Innamorati, M., Lester, D., Balsamo, M., Erbuto, D., Ricci, F., Amore, M., Girardi, P., and Pompili, M. (2014). Factor validity of the Beck Hopelessness Scale in Italian medical patients. J. Psychopathol. Behav. Assess. 36, 300-307.

65. Capanna, C., Stratta, P., Collazzoni, A., D’Ubaldo, V., Pacifico, R., Di Emidio, G., Ragusa, M., and Rossi, A. (2013). Social connectedness as resource of resilience: Italian validation of the Social Connectedness Scale-Revised. J. Psychopathol. 19, 320-326.

66. Lee, R.M., and Robbins, S.B. (1995). Measuring belongingness: The social connectedness and the social assurance scales. J. Couns. Psychol. 42, 232.

67. Soraci, P., Ferrari, A., Abbiati, F.A., Del Fante, E., De Pace, R., Urso, A., and Griffiths, M.D. (2020). Validation and psychometric evaluation of the Italian version of the Fear of COVID-19 Scale. Int. J. Ment. Health Addict., 1-10.

68. Ahorsu, D.K., Lin, C.-Y., Imani, V., Saffari, M., Griffiths, M.D., and Pakpour, A.H. (2020). The fear of COVID-19 scale: development and initial validation. Int. J. Ment. Health Addict., $1-9$

69. Pedrabissi, L., and Santinello, M. (1989). Verifica della validità dello STAl forma Y di Spielberger. Giunti Organ. Spec.

70. Spielberger, C.D. (1983). State-trait anxiety inventory for adults.

71. Smith, J.C. (2010). Smith relaxation states inventory 3 (SRSI3). Raleigh, NC Lulupress.

72. McCabe, R.E. (2015). Subjective units of distress scale. Phobias 18, 361.

73. Singer, J.D., Willett, J.B., and Willett, J.B. (2003). Applied longitudinal data analysis: Modeling change and event occurrence (Oxford university press).

74. Luke, S.G. (2017). Evaluating significance in linear mixed-effects models in R. Behav. Res. Methods 49, 1494-1502.

75. Bates, D., Mächler, M., Bolker, B., and Walker, S. (2014). Fitting linear mixed-effects models using Ime4. arXiv Prepr. arXiv1406.5823.

76. Bolker, B.M. (2008). Ecological models and data in R (Princeton University Press).

77. Kuznetsova, A., Brockhoff, P.B., and Christensen, R.H.B. (2017). ImerTest package: tests in linear mixed effects models. J. Stat. Softw. 82, 1-26.

78. Lenth, R., and Lenth, M.R. (2018). Package 'Ismeans.' Am. Stat. 34, 216-221.

79. Cipolletta, S., and Ortu, M.C. (2020). COVID-19: common constructions of the pandemic and their implications. J. Constr. Psychol., 1-17.

80. Ettman, C.K., Abdalla, S.M., Cohen, G.H., Sampson, L., Vivier, P.M., and Galea, S. (2020). Prevalence of depression symptoms in US adults before and during the COVID-19 pandemic. JAMA Netw. open 3, e2019686-e2019686.

81. Bueno-Notivol, J., Gracia-García, P., Olaya, B., Lasheras, I., López-Antón, R., and Santabárbara, J. (2021). Prevalence of depression during the COVID-19 outbreak: A meta-analysis of community-based studies. Int. J. Clin. Heal. Psychol. 21, 100196.

82. Fiorillo, A., Sampogna, G., Giallonardo, V., Del Vecchio, V., Luciano, M., Albert, U., Carmassi, C., Carrà, G., Cirulli, F., and Dell'Osso, B. (2020). Effects of the lockdown on the mental health of the general population during the COVID-19 pandemic in Italy: Results from the COMET collaborative network. Eur. Psychiatry 63.

83. Poletti, B., Tagini, S., Brugnera, A., Parolin, L., Pievani, L., Ferrucci, R., Compare, A., and Silani, V. (2020). Telepsychotherapy: a leaflet for psychotherapists in the age of COVID19. A review of the evidence. Couns. Psychol. Q., 1-16.

84. Bohil, C.J., Alicea, B., and Biocca, F.A. (2011). Virtual reality in neuroscience research and therapy. Nat. Rev. Neurosci. 12, 752-762.

85. Salisbury, J.P., Aronson, T.M., and Simon, T.J. (2020). At-home self-administration of an immersive virtual reality therapeutic game for post-stroke upper limb rehabilitation. In $\mathrm{CH}$ PLAY 2020 - Extended Abstracts of the 2020 Annual Symposium on Computer-Human Interaction in Play (Association for Computing Machinery, Inc), pp. 114-121. 
86. Donker, T., Klaveren, C. van, Cornelisz, I., Kok, R.N., and Van Gelder, J.-L. (2020). Analysis of usage data from a self-guided app-based virtual reality cognitive behavior therapy for acrophobia: a randomized controlled trial. J. Clin. Med. 9, 1614.

Figure 1. The "Secret Garden", a $360^{\circ}$ Virtual Reality scenario where participants are immersed in a naturalistic and safe digital place, far from the stressful situations experienced in routine daily contexts, in which they can learn how to relax and reflect upon their experience following a guided protocol

\section{List of Table}

Table 1. Demographic characteristics of participants

Table 2. Descriptive statistics for the outcome variables by time points ( 7 days before the start of the intervention - Waiting Period, before the start of the intervention, Day 0 - T0; end of the intervention, Day 7 - T1; 2-week follow-up, Day 21 - T2). Data are provided in means and Standard Deviation (SD).

Table 3. Bonferroni adjusted-pairwise comparisons for all primary and secondary outcome measure across the different time points. ( 7 days before the start of the intervention - Waiting Period, before the start of the intervention, Day 0 - T0; end of the intervention, Day 7-T1; 2-week follow-up, Day 21 - T2). Data are provided in means and Standard Deviation (SD). All df=115.

Table 4. Descriptive statistics for the state outcome measures collected each day during the waiting week ( 7 days before the start of the intervention) and during the intervention phase, after each treatment module. Data are provided in means and Standard Deviation (SD).

\section{Supplementary Material}

Table S1 Estimated parameters for Linear Mixed Models. For all variables, the first model (model 1) was run according to the formula= Outcome $\sim=\sim 1 \mid$ ID; the second model (model 2) was run according to the following formula $=$ Outcome $\sim$ Time $=\sim 1 \mid$ ID, and the third model (model 3) according to this formula $=$ Outcome $\sim$ Time ${ }^{\star}$ Immersion $=\sim 1 \mid$ ID.

Table S2. Estimated parameters for Linear Mixed Models. For all variables, the first model (model 1 ) was run according to the formula= Outcome $\sim=\sim 1 \mid$ ID; the second model (model 2) was run according to the following formula $=$ Outcome $\sim$ Time $=\sim 1 \mid$ ID, and the third model (model 3) according to this formula $=$ Outcome $\sim$ Time ${ }^{*}$ Immersion $=\sim 1 \mid$ ID.

\section{Author Contributions:}

Conception or design of the work: GR, SS, LB; acquisition, analysis, or interpretation of data : GR, GC, AC, DDL, EP, CM, BW, SS; development of VR material: LB, FS, have drafted the work or substantially revised it: GR, DDL, SS, All authors have approved the submitted version and agree to be personally accountable for the author's own contributions.

\section{Competing interests:}

LB is the CEO of Become Hub. FB is a psychological consultant for Become Hub. Become Hub is a company specialized in the development of customized Virtual Reality contents and environments for researchers and neuroscientists. 


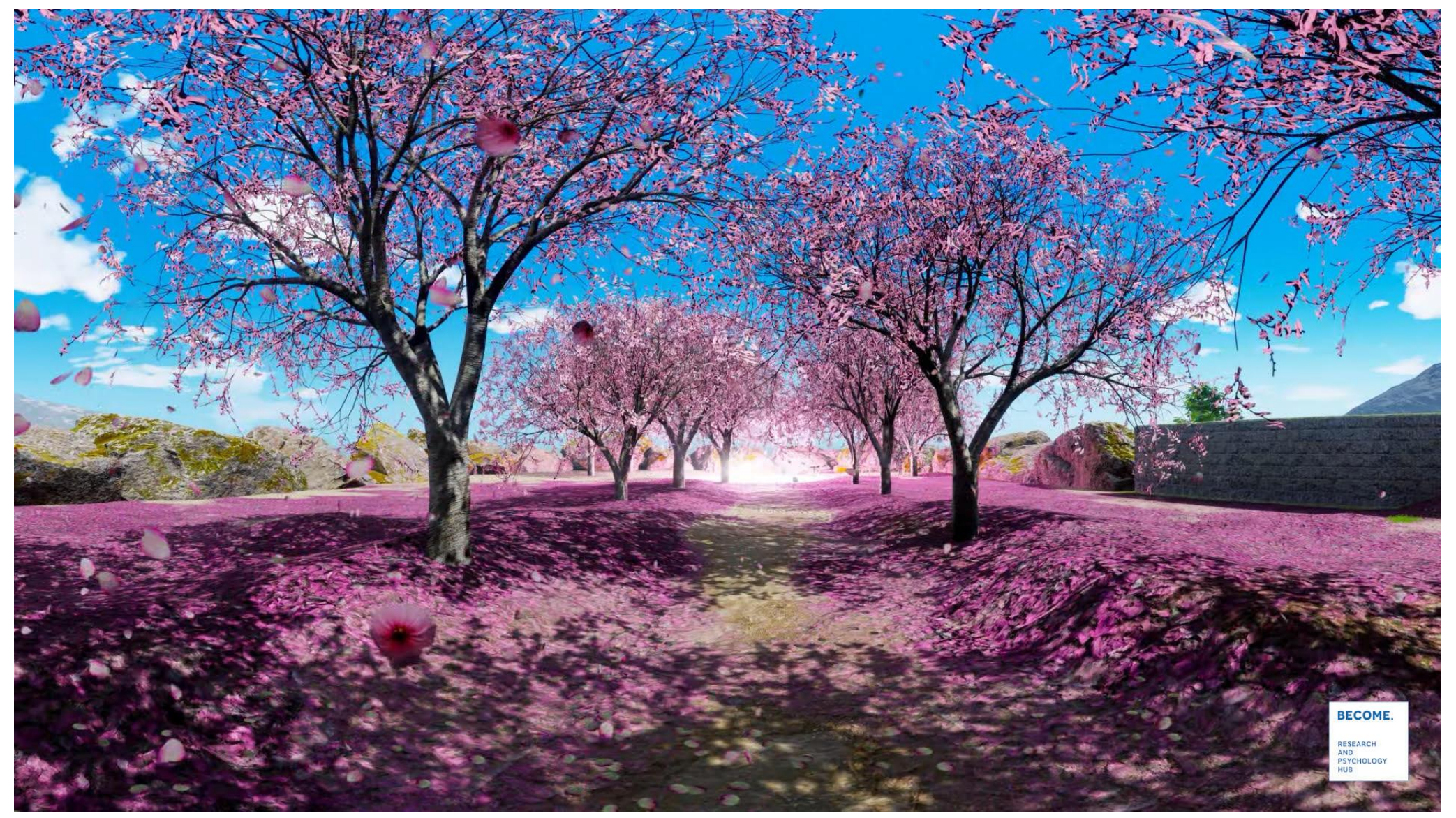


Table 1. Demographic characteristics of participants

\begin{tabular}{lc}
\hline Age (years), mean (SD) & $30.3(12.0)$ \\
Gender, N (\%) & $15(37.5)$ \\
Male & $25(62.5)$ \\
Female & $29(72.5)$ \\
Relationship Status, N (\%) & $10(25)$ \\
Single & $1(2.5)$ \\
Married & $22(55)$ \\
Divorced or others & $18(45)$ \\
Employment Status, N (\%) & $2(5)$ \\
Student & $6(15)$ \\
Employed & $5(12.5)$ \\
Housing arrangements, N (\%) & $23(57.5)$ \\
Living alone & $2(5)$ \\
Living with partner & $2(5)$ \\
Living with partner and children &
\end{tabular}


Immersive modality

Non-Immersive modality

$18(45)$

$22(55)$ 
Table 2. Descriptive statistics for the outcome variables by time points ( 7 days before the start of the intervention - Waiting Period, before the start of the intervention, Day 0 - T0; end of the intervention, Day 7 - T1; 2-week follow-up, Day 21 - T2). Data are provided in means and Standard Deviation (SD).

\begin{tabular}{lcccc}
\multicolumn{1}{c}{ Primary outcome measures } & Waiting Period & T0 & T1 & T2 \\
\hline \multicolumn{1}{c}{} & & & & \\
\hline DASS-21_DEPRESSIONE & 8.75 & 8.85 & 5.65 & 6.37 \\
SD & 6.80 & 8.18 & 5.51 & 4.21 \\
DASS-21_ANSIA & 4.30 & 5.15 & 3.45 & 4.51 \\
SD & 3.88 & 4.77 & 4.51 & 11.7 \\
DASS-21_STRESS & 14.4 & 14.8 & 10.7 & 7.01 \\
Standard deviation & 8.25 & 8.14 & 9.10 \\
DASS-21_GENERAL_DISTRESS & 13.7 & 14.4 & 9.90 & 11.3 \\
Standard deviation & 7.85 & 9.34 & 7.08 & 9.56 \\
PPS-PERCEIVED STRESS & 16.6 & 17.0 & 14.3 & 14.5 \\
Standard deviation & 5.82 & 6.18 & 5.98 & 6.97 \\
BHS- HOPELESSNESS & 4.42 & 4.03 & 3.27 & 4.13 \\
Standard deviation & 3.22 & 3.08 & 2.79
\end{tabular}




\begin{tabular}{lcccc} 
& Waiting Period & T0 & T1 & T2 \\
\hline \multicolumn{1}{c}{ Secondary outcome measures } & & & & \\
\hline SCS- SOCIAL CONNECTEDNESS & 37.4 & 37.4 & 40.1 & 40.1 \\
Standard deviation & 7.81 & 6.63 & 6.64 & 6.96 \\
FCOR- FEAR OF CORONAVIRUS & 24.6 & 23.8 & 22.9 & 23.0 \\
Standard deviation & 4.62 & 4.74 & 5.00 & 5.36 \\
\hline
\end{tabular}

Table 3. Bonferroni adjusted-pairwise comparisons for all primary and secondary outcome measure across the different time points. (7 days before the start of the intervention - Waiting Period, before the start of the intervention, Day 0 - T0; end of the intervention, Day 7 - T1; 2-week follow-up, Day $21-\mathrm{T} 2$ ). Data are provided in means and Standard Deviation (SD). All df=115. 


\begin{tabular}{|c|c|c|c|c|c|c|}
\hline & Contrasts & & Estimate & $\begin{array}{l}\text { Standard Error } \\
\text { (SE) }\end{array}$ & $t$-ratio & P-value \\
\hline \multirow[t]{6}{*}{ DASS-21 Depression } & Waiting Period & T0 & 0.100 & 0.908 & 0.629 & 1.0000 \\
\hline & & $\mathrm{T} 1$ & -3.100 & 0.908 & -3.413 & 0.0053 \\
\hline & & T2 & -2.519 & 0.924 & -2.727 & 0.0443 \\
\hline & $\mathrm{T} 1$ & T0 & 3.200 & 0.908 & 3.523 & 0.0037 \\
\hline & & T2 & 0.581 & 0.924 & -2.727 & 0.0053 \\
\hline & T0 & T2 & 2.619 & 0.924 & 2.836 & 0.324 \\
\hline \multirow[t]{6}{*}{ DASS-21 Stress } & Waiting Period & TO & 0.350 & 1.14 & 0.308 & 1.000 \\
\hline & & $\mathrm{T} 1$ & -3.7000 & 1.14 & -3.257 & 0.0089 \\
\hline & & T2 & -2.878 & 1.16 & -2.491 & 0.0849 \\
\hline & T1 & T0 & 4.050 & 1.14 & 3.565 & 0.0032 \\
\hline & & T2 & 0.822 & 1.16 & -2.491 & 0.0849 \\
\hline & TO & T2 & 3.228 & 1.16 & 2.794 & 0.0365 \\
\hline \multirow[t]{3}{*}{ DASS-21 General Distress } & Waiting Period & T0 & 0.65 & 1.14 & 0.570 & 1.0000 \\
\hline & & T1 & -3.83 & 1.14 & -3.352 & 0.0065 \\
\hline & & T2 & -2.70 & 1.16 & -2.328 & 0.1300 \\
\hline
\end{tabular}




$\begin{array}{lllllll} & \text { T1 } & \text { T0 } & 4.47 & 1.14 & 3.921 & 0.0009 \\ \text { DASS-21 PPS } & & \text { T2 } & 1.12 & 1.16 & 0.968 & 1.0000 \\ & \text { TO Waiting Period } & \text { T2 } & 3.35 & 1.16 & 2.888 & 0.0278 \\ & & \text { T0 } & 0.475 & 0.869 & 0.547 & 1.0000 \\ & & \text { T1 } & -2.225 & 0.869 & -2.561 & 0.0704 \\ \text { DASS-21 BHS } & \text { T1 } & -2.114 & 0.883 & -2.393 & 0.1101 \\ & & \text { T0 } & 2.7000 & 0.869 & 3.107 & 0.0143 \\ & \text { TO } & \text { T2 } & 0.111 & 0.883 & 0.126 & 1.0000 \\ & \text { Waiting Period } & \text { T2 } & 2.589 & 0.883 & 2.930 & 0.0245 \\ & & \text { T0 } & -0.4000 & 0.313 & -1.277 & 1.0000 \\ & & \text { T1 } & -1.1500 & 0.313 & -3.670 & 0.0022 \\ \text { DASS-21 SCS } & \text { T2 } & -0.3296 & 0.319 & -1.034 & 1.0000 \\ & \text { T1 } & \text { T0 } & 0.7500 & 0.313 & 2.393 & 0.1098 \\ & & \text { T2 } & 0.8204 & 0.319 & 2.574 & 0.0679 \\ & \text { TO } & \text { T2 } & -0.0704 & 0.319 & -0.221 & 1.0000 \\ & \text { Waiting Period } & \text { T0 } & 0.0250 & 0.944 & 0.026 & 1.0000 \\ & & \text { T1 } & 2.7000 & 0.944 & 2.859 & 0.0303 \\ & & \text { T2 } & 2.7832 & 0.960 & 2.899 & 0.0269 \\ & & \text { T0 } & -2.6750 & 0.944 & -2.833 & 0.0327\end{array}$




$\begin{array}{lllllll} & & \text { T2 } & 0.0832 & 0.960 & 0.087 & 1.0000 \\ \text { DASS-21 FCOR } & \text { TO } & \text { T2 } & -2.7582 & 0.960 & -2.863 & 0.0291 \\ & \text { Waiting Period } & \text { T0 } & -0.800 & 0.476 & -1.680 & 0.5738 \\ & & \text { T1 } & -1.675 & 0.476 & -3.518 & 0.0037 \\ & & \text { T2 } & -1.478 & 0.484 & -3.051 & 0.0170 \\ \text { T1 } & \text { T0 } & 0.875 & 0.476 & 1.838 & 0.4121 \\ & \text { T2 } & 0.197 & 0.484 & 0.407 & 1.0000 \\ & \text { TO } & \text { T2 } & 0.678 & 0.484 & 1.399 & 0.9864\end{array}$

Table 4. Descriptive statistics for the state outcome measures collected each day during the waiting week (7 days before the start of the intervention) and during the intervention phase, after each treatment module. Data are provided in means and Standard Deviation (SD). 
Waiting Period

Intervention Week

\begin{tabular}{lrc}
\hline \multicolumn{1}{c}{ State outcome measures } & \\
\hline STAI-STATE & 42.0 & 35.3 \\
SD & 9.48 & 7.52 \\
SMITH3- REST/REFRESHED & 3.24 & 3.67 \\
SD & 0.693 & 0.877 \\
SMITH3-ENERGIZED & 3.09 & 3.46 \\
SD & 0.604 & 1.000 \\
SMITH3- PHYSICAL RELAXATION & 2.22 & 3.15 \\
SD & 0.871 & 1.25 \\
SMITH3- AT EASE/PEACE & 3.20 & 4.76 \\
SD & 0.794 & 4.41 \\
SMITH3- JOY & 3.38 & 3.61 \\
SD & 0.907 & 1.02
\end{tabular}


Waiting Period

$$
\text { SMITH3- MENTAL_QUIET }
$$

SD

SMITH3- AWARE

SD

SMITH3- SOMATIC DISTRESS

SD

SMITH3- EMOTIONAL DISTRESS

SD

SMITH3- SOMATIC DISTRESS

SD

SUDS

$\begin{array}{rr}2.50 & 3.31 \\ 0.805 & 0.964 \\ 3.06 & 3.25 \\ 1.01 & 0.961 \\ 1.66 & 1.40 \\ 0.549 & 0.498 \\ 1.76 & 1.48 \\ 0.661 & 0.460 \\ 2.01 & 1.62 \\ 0.833 & 0.667 \\ 17.6 & 10.7\end{array}$

